\title{
Growth and Characterization of Eu-Cu-O Thin Films on YSZ(100) Substrates
}

\author{
J. Gao, W.H. Tang and C.Y. Yau
}

\begin{abstract}
High quality epitaxial $\mathrm{Eu}_{2} \mathrm{CuO}_{4}$ (ECO) thin films have been deposited on yttrium-stabilized zirconia (YSZ) (100) substrates by magnetron rf sputtering method. The obtained ECO films are highly $c$-axis oriented and well crystallized, as shown by $x$-ray diffraction, rocking curves. The influence of oxygen out-diffusion on the c-axis length and the resistance behavior were studied. The post vacuum annealing removes the excess oxygen from the structure of ECO. As a result, the $c$-axis length decreases and the resistivity increases. By using $\mathrm{ECO}$ as a buffer layer, extremely smooth surface of YBCO was obtained on YSZ substrates.
\end{abstract}

Index Terms-High Temperature Superconductors, Materials Processing, Sputtering, Superconducting Films

\section{INTRODUCTION}

$\mathrm{T}_{\mathrm{s}}$ o obtain epitaxial high- $T_{c}$ superconducting thin films, selection of suitable substrates is of great importance. A suitable substrate normally means that it has good structural and chemical compatibilities with the desired thin film, meanwhile its physical properties must not hamper the application purpose of the obtained thin film. Unfortunately, the perfect substrate for the epitaxial growth of high- $T_{c}$ superconducting thin films is very rare. Thus, buffer layers are generally used to improve the initial growth of the thin films on those substrates having poor structural and chemical compatibilities with high- $T_{c}$ thin films, such as $\mathrm{Si}, \mathrm{Al}_{2} \mathrm{O}_{3}$ and yttrium-stabilized zirconia (YSZ) substrates. For example, YSZ substrate, its lattice parameters $(a / \sqrt{2} \sim 3.63 \AA)$ are quite different from those of $\mathrm{YBa}_{2} \mathrm{Cu}_{3} \mathrm{O}_{7-\delta}$ (YBCO) $(a \sim 3.82 \AA, b \sim$ $3.88 \AA$ ). Furthermore, an intermediate $\mathrm{BaZrO}_{3}$ is often formed due to the diffusion of $\mathrm{Ba}$ into YSZ substrate [1,2]. Thus various buffer layers, such as $\mathrm{PrBa}_{2} \mathrm{Cu}_{3} \mathrm{O}_{\mathrm{y}}$ (PBCO) [3] and $\mathrm{Nd}_{2} \mathrm{CuO}_{4}[4]$, have been used for growing thin film of YBCO. Unlike $\mathrm{PrBa}_{2} \mathrm{Cu}_{3} \mathrm{O}_{\mathrm{y}}$, ECO shows higher stabilities in crystal structure and has no structural transition. $\mathrm{Nd}_{2} \mathrm{CuO}_{4}(a \sim 3.94 \AA)$ with a 214-T' structure, had been successfully used as a buffer for making ultra-thin films of YBCO on YSZ [4]. Compared with that of $\mathrm{Nd}_{2} \mathrm{CuO}_{4}$, the lattice parameter of ECO $(a \sim$

Manuscript received August 6,2000. This work was supported by the Research Grants Council (RGC) of Hong Kong and the Committee on research and conference grants (CRCG) of the University of Hong Kong.

J. Gao is with Department of Physics, The University of Hong Kong, Pokfulam Road, Hong Kong (telephone: 852-28597948, fax: 852-25599152, e-mail: jugao@hku.hk).

W.H. Tang is with Department of Physics, The University of Hong Kong, Pokfulam Road, Hong Kong (telephone: 852-28592195, fax: 852-25599152, e-mail: whtang@hku.hk).

C.Y. Yau is with Department of Physics, The University of Hong Kong, Pokfulam Road, Hong Kong
$3.89 \AA$ ) matches better with those of $\mathrm{YBa}_{2} \mathrm{Cu}_{3} \mathrm{O}_{7-\delta}$. Thus, ECO could be a good buffer layer for the growth of high- $T_{c}$ superconducting thin films. In this work, ECO thin films have been grown on YSZ (100) substrates by magnetron if sputtering method. The films were investigated by $\mathrm{x}$-ray diffraction, rocking curves, surface step profile, AFM and SEM. The changes in the $c$-axis length and the resistance behavior were studied on the films treated by post vacuum annealing.

\section{EXPERIMENTAL}

ECO monolayer and ECO/YBCO bilayer thin films were deposited on YSZ (100) substrates by off-axis rf sputtering[5]. The depositing temperature referred hereafter to as the substrate temperature $T_{s}$ was measured using a thermocouple inserted into the stainless steel substrate heater. The substrate was stuck on the heater by silver paste. The substrate temperature was $750^{\circ} \mathrm{C}$ to $770^{\circ} \mathrm{C}$. The depositing gas was a mixture of argon and oxygen with different pressure ratios $\left(P_{A r} / P_{O 2} \sim 3\right.$ to 4$)$. The $\mathrm{x}$-ray diffraction was performed on the Siemens D5000 x-ray diffractometer. The crystallinities of ECO thin films were examined by measuring the rocking curve of the (006) peak. To study the influence of oxygen out-diffusion on the $c$-axis length and the resistance behavior, post vacuum annealing for selected ECO thin films was performed.

The film thickness and the surface roughness were measured by using the Dektak3ST surface profiler. The vertical resolution is $1 \AA$ for the measuring range of $65 \mathrm{k} \AA$. The scanning length is larger than $1000 \mu \mathrm{m}$. The surface morphologies of ECO thin films were examined by a Cambridge 440 scanning electron microscope (SEM) and atomic force microscopy (AFM). Micro-bridges $(50 \mu \mathrm{m}$ in width and $200 \mu \mathrm{m}$ in length) were made by photolithography for the measurement of the resistivity.

\section{RESULTS AND DISCUSSIONS}

X-ray diffraction has been performed on ECO thin films to examine their crystallinities. Fig. 1 shows one typical $x$-ray diffraction pattern of an ECO thin film. It is found that only (00l) $(l=2 n)$ diffraction peaks for ECO were observed, indicating a highly $c$-axis orientation. The insert plot of the figure gives a rocking curve of the (006) peak for ECO. The rocking curve indicates a very small value of the full width at half maximum (FWHM), typically smaller then $0.15^{\circ}$. This value even is smaller than that of the (005) peak with lower $2 \theta$ value $\left(\sim 38.5^{\circ}\right)$ for YBCO. Very small FWHM value suggests good crystallinity of ECO films. 


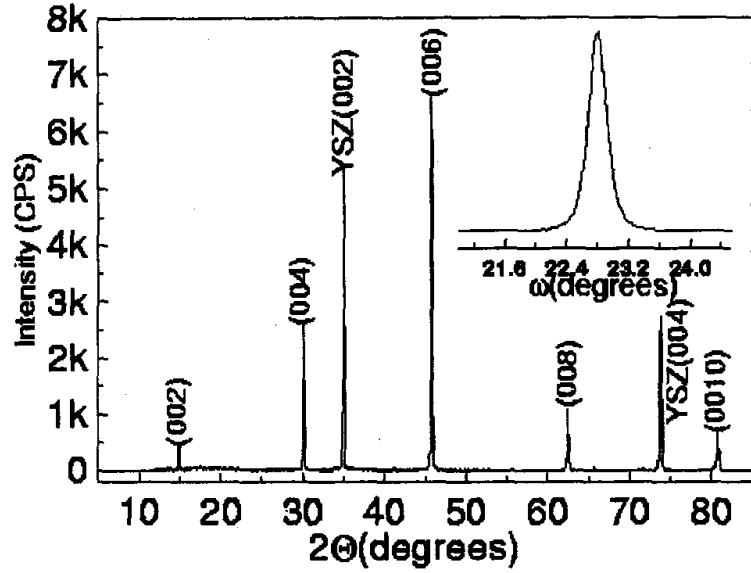

Fig. 1 Typical x-ray diffraction pattern for the ECO thin film on YSZ(100) substrate. The insert shows the rocking curves of (006) peak for ECO.

The ECO thin film is very shiny and shows a good surface. The average roughness of an ECO thin film is less than $10 \mathrm{~nm}$ over a wide scanning region $(1000 \mu \mathrm{m})$. Unlike 123-phase thin films, ECO thin films exhibit very smooth surface without outgrowths. That could be the common feature for the 214-phase thin films.[6,7] It could be attributed to their high structural stability.

The ECO thin films exhibit semiconducting behavior. Fig.2 shows the temperature dependences of the resistivities of ECO and $\mathrm{PBCO}$ thin films. It is seen that the resistivity of ECO is much higher than that of PBCO, especially at lower temperature. For example, at $77 \mathrm{~K}$, the resistivities for ECO and $\mathrm{PBCO}$ are $1407 \mathrm{~m} \Omega . \mathrm{cm}$ and $7 \mathrm{~m} \Omega . \mathrm{cm}$, respectively. Also, the increasing rate of the resistivity with temperature for $\mathrm{ECO}$ thin films is also remarkably larger than that of PBCO. PBCO has been used as a common bufferlayer and barrier material for high $T_{c}$ junctions. However, it has several drawbacks, such as: poor surface, structural instability, chemical interdiffusion and relatively low resistivity. Compared with $\mathrm{PBCO}, \mathrm{ECO}$ is superior over PBCO for the applications as a buffer layer or a barrier material.

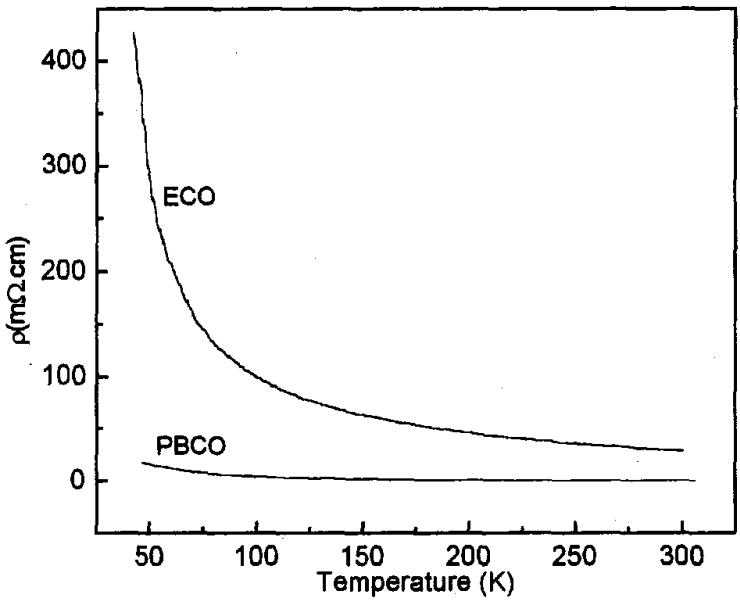

Fig. 2 Temperature dependences of the resistivities of ECO and PBCO thin films.

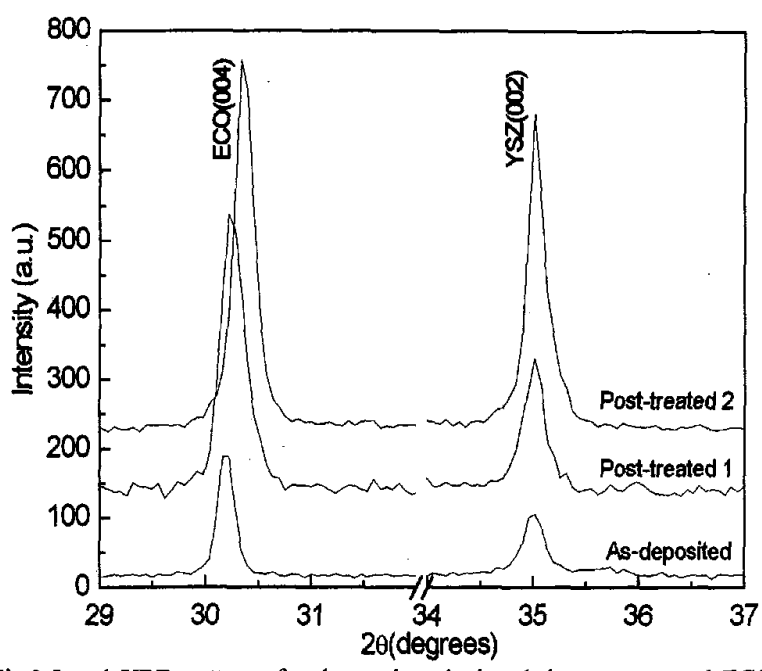

Fig.3 Local XRD patterns for the as-deposited and the post- treated ECO films. Post-treated 1 and 2 correspond to the films annealed at $400^{\circ} \mathrm{C}$ and $500^{\circ} \mathrm{C}$, respectively.

To test the structural stability of ECO with the change of oxygen content, we have investigated the influence of oxygen out-diffusion on the $c$-axis length and the resistance behavior of ECO thin film was studied by post vacuum annealing. The ECO thin film was stuck by the silver paint onto the heater and then put it into the vacuum chamber $\left(\sim 10^{-4} \mathrm{mbar}\right)$. The ECO film was annealed at $400^{\circ} \mathrm{C}$ and $500^{\circ} \mathrm{C}$ for 30 minutes, respectively. Fig. 3 shows the local XRD patterns for the as-deposited and the post annealed ECO films. It is found that the (004) peak of ECO was shifted to high $2 \theta$ angle and the $(002)$ peak of YSZ remained unchanged. In other words, the post vacuum annealing reduced the c-axis parameter of ECO. Vacuum annealing removes excess oxygen from the structure of ECO, which results in the reduction of the $c$-axis length. No structural transition was observed after the vacuum annealing. The variation of the $c$-axis length for ECO with oxygen is different from the case of 123-phase materials.[8]

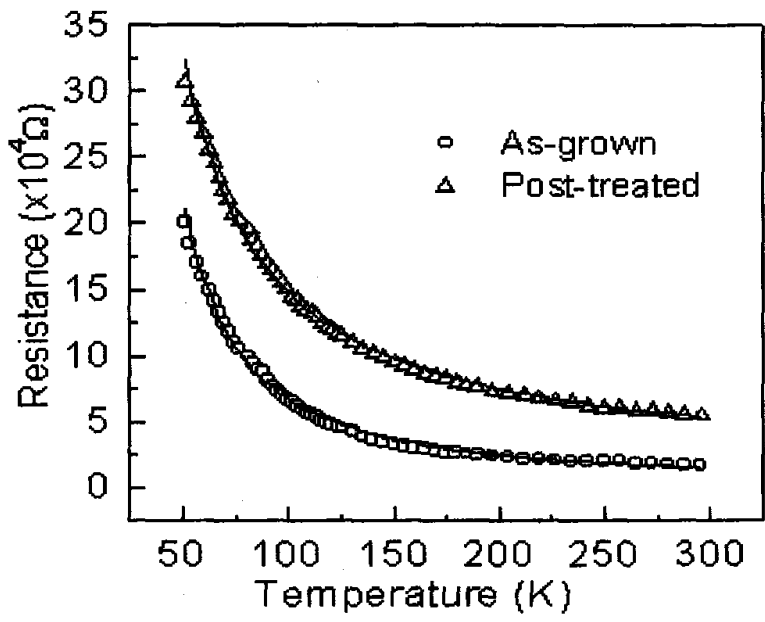

Fig. 4 R-T curves for the as-grown and the post vacuum annealed at $400^{\circ} \mathrm{C}$ for 30 minutes ECO thin films. The lines represent the fitting results by a 3D VRH mechanism. 


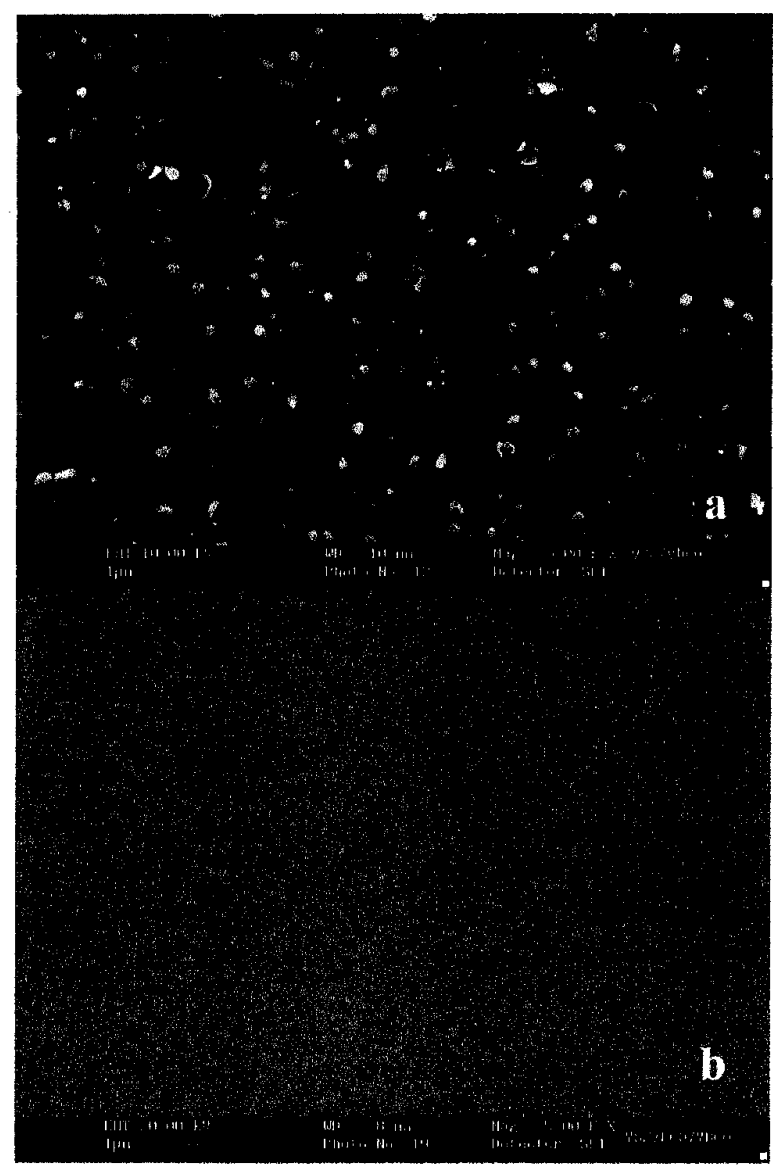

Fig.5 The SEM surface morphology photos for the YBCO thin films (a) without and (b) with $\mathrm{ECO}$ buffer layer. The thickness of YBCO thin film is $\sim 160 \mathrm{~nm}$, and the thickness of ECO buffer layer is $\sim 40 \mathrm{~nm}$.

Fig.4 shows the R-T curves for the as-grown and the post vacuum annealed $\mathrm{ECO}$ thin films. The lines represent the fitting curves. The oxygen out-diffusion results in an increase of the resistance. Like high $T_{c}$ superconducting cuprates, oxygen content is one of factors, which effect the carrier concentration for ECO. The experimental data can be fitted by

$R=R_{0} \exp \left(T_{0} / T\right)^{1 / 4}$

where $T_{0}$ is a characteristic temperature. The excellent fitting results suggest that the resistance behavior can be well described by a $3 \mathrm{D}$ variable-range-hopping $(\mathrm{VRH})$ conduction mechanism, which is similar with $\mathrm{PBCO}$ [9].

As mentioned above, ECO thin films have good crystallinity, stable crystal structure, excellent surface and high resistivity. Also, the in-plane lattice parameters match well with that of YBCO. Those features meet the requirements for a buffer layer and a barrier material. We have grown YBCO thin films on YSZ substrates by using ECO as a buffer layer. The obtained YBCO thin films exhibit very smooth surface. No outgrowths were observed. Fig.5 shows the SEM surface morphology photos for the YBCO thin films (a) without and (b) with ECO buffer layer. The thickness of YBCO thin film is $\sim 160 \mathrm{~nm}$, and the thickness of ECO buffer layer is $\sim 40 \mathrm{~nm}$. It can be seen that YBCO thin film grown directly on YSZ substrate shows poor surface morphology. Many small outgrowths were observed, which is very common for 123-phase thin films. By using the ECO buffer layer, the outgrowth was totally eliminated. The YBCO thin film with ECO buffer layer has an extremely smooth surface. The average roughness is less than $5 \mathrm{~nm}$ measured by surface step profiler. Such smooth surface could result from the layer-by-layer growth mechanism. It has been reported that the growth of YBCO thin film on YSZ substrates can change from island formation to layer-by-layer growth by using $\mathrm{La}_{1.85} \mathrm{Sr}_{0.15} \mathrm{CuO}_{4}$ as a buffer layer.[6]

\section{CONCLUSION}

We have fabricated high quality epitaxial $\mathrm{Eu}_{2} \mathrm{CuO}_{4}$ thin films on yttrium-stabilized zirconia (100) substrates by magnetron rf sputtering method. The obtained ECO films are highly $c$-axis oriented and well crystallized, as shown by $\mathrm{x}$-ray diffraction and rocking curves. The changes in the c-axis length and the resistance behavior were studied on the films treated by post vacuum annealing. The post vacuum annealing removes the excess oxygen from the structure of ECO. As a result, the $c$-axis length decreases and the resistivity increases. By using ECO as a buffer layer, extremely smooth surfaces of YBCO thin films were obtained on YSZ substrates and the growth of YBCO could change from island mode to layer-by-layer one.

\section{ACKNOWLEDGMENT}

The SEM measurement was made in the Microscope Unit of the University of Hong Kong. Authors thank Dr. K.Y. Chan (Department of Chemistry, The University of Hong Kong) for his help in AFM experiments.

\section{REFERENCES}

[1] J.G. Wen, C. Traeholt, H.W. Zandbergen, K. Joosse, E. M. C. M. Reuvekamp, and H. Rogalla "A HREM study of the atomic structure and the growth mechanism at the $\mathrm{YBa} 2 \mathrm{Cu} 307 / \mathrm{YSZ}$ interface", Physica C, vol.218, pp.29-42, 1993

[2] J. Gao, W.H. Wong, and J. Xhie, "Formation of outgrowths at the initial growing stage of $\mathrm{YBa} 2 \mathrm{Cu} 3 \mathrm{Ox}$ ultrathin films on $\mathrm{ZrO} 2$ substrates", Appl. Phys. Lett. vol.67, pp.2232-2234, 1995

[3] T. Terashima, K. Shimura, Y. Bando, Y. Matsuda, A. Fujiyama, and S. Komiyama,"Superconductivity of one-unit-cell thick $\mathrm{YBa}_{2} \mathrm{Cu}_{3} \mathrm{O}_{7}$ thin film", Phys. Rev. Lett., vol.67, pp.1362-1365, 1991

[4] J. Gao, T.C. Chui and W.H. Tang, "Ultrathin films of YBaCuO grown on YSZ substrates with a new buffer layer Nd-Cu-O", IEEE Trans. on Appl. Supercond. 9 (1999) 1661

[5] J. Gao, B. Häuser, H. Rogalla, "High critical current density ultrathin $\mathrm{YBa}_{2} \mathrm{Cu}_{3} \mathrm{O}_{\mathrm{x}}$ films made by a modified rf-magnetron sputtering technique", J. Appl. Phys., vol.67, pp2512-2515, 1990

[6] J. Gao, G. J. Lian and G. C. Xiong, "Improved initial epitaxial growth of superconducting $\mathrm{YBa}_{2} \mathrm{Cu}_{3} \mathrm{O}_{7}$ thin films on $\mathrm{Y}-\mathrm{ZrO}_{2}$ substrates with a $\mathrm{La}_{1.85} \mathrm{Sr}_{0,15} \mathrm{CuO}_{4}$ buffer layer", Physica C, vol.330, pp. 160-164, 2000

[7] J. Gao, W.H. Tang, and T.C. Chui, "Enhanced initial epitaxy of $\mathrm{YBa}_{2} \mathrm{Cu}_{3} \mathrm{O}_{\mathrm{y}}$ ultrathin films grown on $\mathrm{YSZ}$ substrates by using a new buffer layer of $\mathrm{Nd}_{2} \mathrm{CuO}_{4}, "$ Physica C, vol.330, pp.33-38, 2000.

[8] W.H. Tang and J. Gao, "The relation between $c$-axis lattice parameter and superconducting transition temperature of $\mathrm{NdBa}_{2} \mathrm{Cu}_{3} \mathrm{O}_{y}$ thin films", Physica C, vol.313, pp.115-120, 2000.

[9] W.H. Tang and J. Gao, "High resistivity of tetragonal $\mathrm{Pr}_{1+\times} \mathrm{Ba}_{2-x} \mathrm{Cu}_{3} \mathrm{O}_{y}$ solid solution", Physica C, vol.315, pp.66-70, 1999. 